OPEN ACCESS

Edited by:

Jose Bargas,

Universidad Nacional Autónoma de

México, Mexico

Reviewed by:

Vikas Mishra,

Babasaheb Bhimrao Ambedkar

University, India

Alfredo Meneses,

Centro de Investigación y de Estudios Avanzados del Instituto Politécnico Nacional (CINVESTAV-IPN), Mexico

*Correspondence:

Jorge H. Medina jmedina@fmed.uba.ar

Received: 28 November 2017 Accepted: 18 January 2018 Published: 06 February 2018

Citation:

Medina JH (2018) Neural, Cellular and Molecular Mechanisms of Active

Forgetting.

Front. Syst. Neurosci. 12:3. doi: 10.3389/fnsys.2018.00003

\section{Neural, Cellular and Molecular Mechanisms of Active Forgetting}

\author{
Jorge H. Medina ${ }^{1,2 *}$ \\ ${ }^{1}$ Laboratorio de Memoria, IBCN Universidad de Buenos Aires-Consejo Nacional de Investigaciones Científicas y Técnicas \\ (UBA-CONICET), Buenos Aires, Argentina, ${ }^{2}$ Departamento de Fisiología, Facultad de Medicina, Universidad de Buenos \\ Aires, Buenos Aires, Argentina
}

The neurobiology of memory formation attracts much attention in the last five decades. Conversely, the rules that govern and the mechanisms underlying forgetting are less understood. In addition to retroactive interference, retrieval-induced forgetting and passive decay of time, it has been recently demonstrated that the nervous system has a diversity of active and inherent processes involved in forgetting. In Drosophila, some operate mainly at an early stage of memory formation and involves dopamine (DA) neurons, specific postsynaptic DA receptor subtypes, Rac1 activation and induces rapid active forgetting. In mammals, others regulate forgetting and persistence of seemingly consolidated memories and implicate the activity of DA receptor subtypes and AMPA receptors in the hippocampus (HP) and related structures to activate parallel signaling pathways controlling active time-dependent forgetting. Most of them may involve plastic changes in synaptic and extrasynaptic receptors including specific removal of GluA2 AMPA receptors. Forgetting at longer timescales might also include changes in adult neurogenesis in the dentate gyrus (DG) of the HP. Therefore, based on relevance or value considerations neuronal circuits may regulate in a time-dependent manner what is formed, stored, and maintained and what is forgotten.

Keywords: memory, forgetting, Drosophila, dopamine, rac 1, hippocampus, BDNF, AMPA receptors

\section{INTRODUCTION}

In general sense forgetting refers to as the inability to express some information acquired previously, no matter whether or not that information establishes a retrievable memory. In strict sense forgetting is referred to as the inability to recall something now that could be retrieved on an earlier occasion. This could happen if an established memory is no longer available. In other words, that memory is lost. In addition, the possibility exists that forgetting is due to a temporary failure in accessing to that memory: in other words, a deficit in memory retrieval. However, forgetting must be differentiated from amnesia. Both have memory loss but while forgetting is a natural process, amnesia is a pathological one.

Two main ideas about forgetting emerged in the field of experimental psychology. One dominant hypothesis postulates that interference at different steps of memory processing is the principal cause of forgetting (Wixted, 2004). This interference could be at encoding, or at the formation and consolidation or even at the moment of retrieving a memory (Skaggs, 1933). For instance, Wixted (2004) suggests that the amnesic effect of a new learning on previously encoded material (retroactive interference) is mainly due to the use of the resources available to consolidate the original trace. The immediate early gene Arc is one of the resources involved in memory competition (Martínez et al., 2012). The second hypothesis postulates that passive or active decay of the memory trace is 
also responsible to forgetting. This idea has been consistently criticized during many years (Neath and Brown, 2012). However, some recent neurobiological data regarding cellular and molecular mechanisms of long-term memory (LTM) persistence and forgetting appears to endorse the idea that active forgetting is an important part in determining the fate of memories (see below). A detailed description of biological and non-biological arguments supporting the idea that the brain is endowed with intrinsic forgetting processes is provided by two recent review articles (Hardt et al., 2013; Davis and Zhong, 2017).

During the last five decades a great body of evidence accumulated regarding the neurobiology of memory formation, and its main consequences extinction and reconsolidation (McGaugh, 2000; Sara, 2000; Kandel, 2001; Dudai, 2002; Morris, 2006). However, much less attention has been focused on the mechanisms of active forgetting (Davis and Zhong, 2017). Without forgetting you may have in mind all the information from the external world, including those data which are not relevant. As the argentinian writer Jorge Luis Borges pointed out in his story "Funes el memorioso" (Funes the memorizer), if one acquires and stores all the information we experience in a given day, we will take almost another day to retrieve it, and therefore we will learn nothing at all in such a day. The sentences "Memories are islands in an ocean of forgetting" and "Memory: name we give to the cracks of stubborn forgetting" are two wonderful, poetic but realistic views of what Borges and many neuroscientists in the last 50 years consider memory and forgetting: forgetting of learned experiences is, at least, as important as the process of making memories.

Although there are several types of forgetting including retroactive interference (Wixted, 2004), retrieval-induced forgetting (Anderson, 2003), passive decay of memory and active or intrinsic forgetting (see the following reviews for more details and references, Frankland et al., 2013; Hardt et al., 2013; Davis and Zhong, 2017), in this review I will focus on what is known as active forgetting. In a general sense active forgetting is the inherent neural, cellular and molecular processes involved in erasing the substrate of a memory or in suppressing its accessibility. From a historical point of view I will first present some indirect evidence for the existence of a natural and active process of forgetting. This experimental evidence is however inconclusive and fragmentary. Therefore, the main focus of this article will be on what it is considered the direct evidence of active inherent forgetting, describing mechanisms of active forgetting in Drosophila and in rodents. Direct evidence is referred to the demonstration of a mechanism or a sequence of events that is required for and sufficient to erase the substrate of a given memory. Finally, some of the processes that modulate the rate of active forgetting will be briefly described.

\section{INDIRECT EVIDENCE FOR ACTIVE FORGETTING}

During the last 15 years a wealth of indirect evidence supports the idea that an inherent active mechanism of forgetting is present in the central nervous system. Studies analyzing the cellular and molecular underpinnings responsible for establishing remote memories and for their persistence shed new light for a better understanding of active forgetting process of consolidated memories (see for references Katche et al., 2013a,b; Bekinschtein et al., 2014; Katche and Medina, 2017). Forgetting of very short-lived memories including the so-called immediate memory (McGaugh, 2000), and also forgetting due to suppression of memory expression are not considered in this review; in addition, in order to avoid confounds between promotion of forgetting with inhibition of memory consolidation or inhibition of forgetting with facilitation of memory consolidation, all the findings described in this section mainly involve specific modifications in the durability of consolidated LTM without modifications in acquisition, memory formation, or retrieval (see also below "predictions and requirements" in direct evidence):

1. Active forgetting of long-lasting contextual fear memory was achieved by late posttraining temporary knocking down of NMDA receptors or alpha-CaMKII activity in the forebrain (Wang et al., 2003; Cui et al., 2004). These findings are consistent with those showing that CaMKII heterozygous knockout mice exhibited rapid forgetting of remote, but not recent LTM (Frankland et al., 2001). Recently, chronic inhibition of the NMDA receptor and L-type voltagedependent $\mathrm{Ca}^{2+}$ channel maintains long-term object location memory that otherwise would have been forgotten (Sachser et al., 2016).

2. Selective forgetting of remote memories was found after manipulation of several protein kinases. For instance, ERK1/2 participates late after training to sustain LTM storage of two different hippocampus (HP)-dependent learning tasks (Bekinschtein et al., 2008; Eckel-Mahan et al., 2008). A circadian oscillation of the phosphorylation state of ERK1/2 in the HP has been implicated in memory persistence (Eckel-Mahan, 2012). Inducible and targeted deletion of hippocampal ERK5 induced a specific impairment in remote avoidance memory (Pan et al., 2012). Shan et al. (2008) demonstrated that knocking down adenylyl cyclases 1 impaired remote contextual fear memory. Adenylyl cyclases 1 and 8 regulate long-lasting transcriptional changes in the HP important for memory persistence (Wieczorek et al., 2010). Most of these genes are up-regulated in wild-type mice $48 \mathrm{~h}$ after training. These authors found that knocking-down adenylyl cyclase 8 provoked active forgetting of remote memory. Finally, selective forgetting of well-consolidated memories was observed after blockade of PKM $\zeta$ (Sacktor, 2011). PKM $\zeta$ appears to keep memory storage by regulating GluA2-dependent AMPA receptor trafficking (Migues et al., 2010). This atypical PKC isoform is persistently activated during L-LTP and was repeatedly suggested to be involved in maintenance of memory storage long after memory is consolidated into LTM (Sacktor, 2011). It has been shown that PKM $\zeta$ maintains object recognition memory for about 1 week preventing the internalization of GluA2-containing AMPA receptors in the dorsal HP (Migues et al., 2010). The inhibition of synaptic removal of these hippocampal receptors supports memory persistence of consolidated object location and food-reward conditioned place preference without altering 


\section{Drosophila}

Aversive and appetitive memories. Timescale in hours after training.

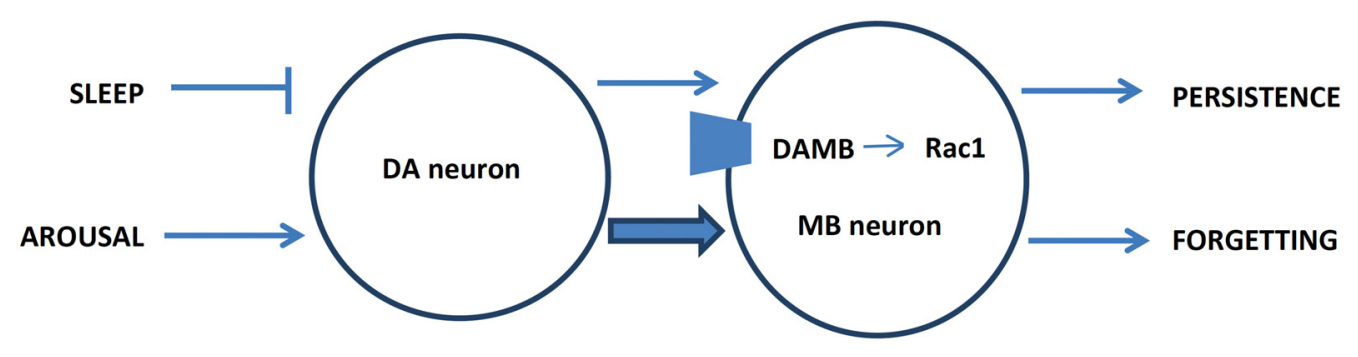

2. Rodent

Recognition and aversive memories. Timescale in hours-few days after training.

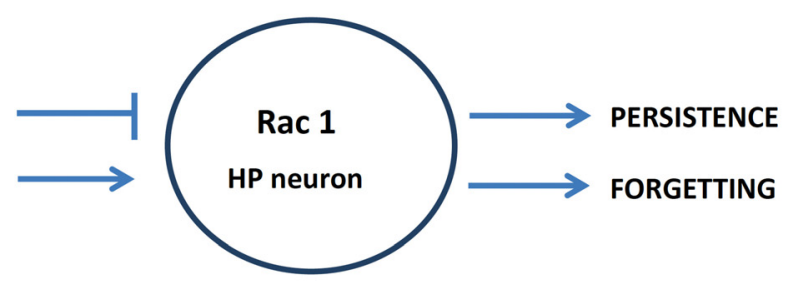

Appetitive memories. Timescale in days after training.

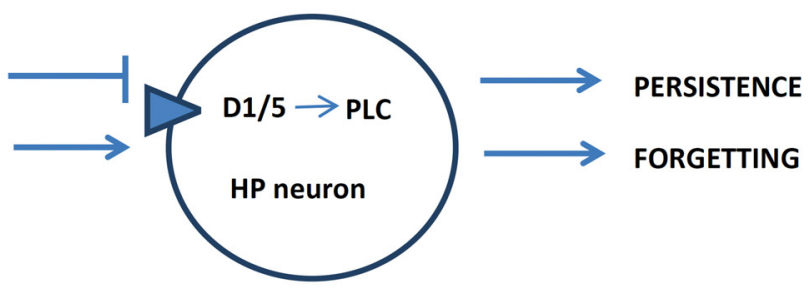

Aversive, appetitive and spatial memories. Timescale in weeks after traning.

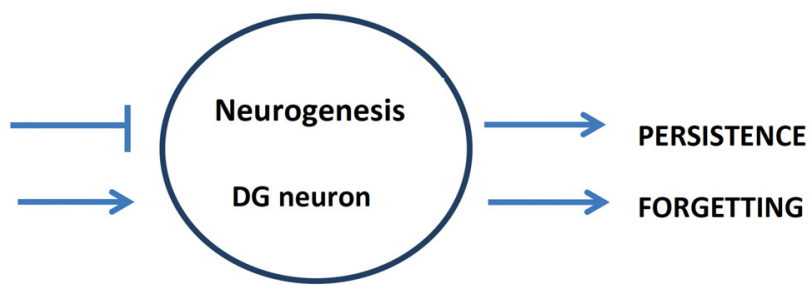

FIGURE 1 | Schematic representation of major mechanisms of active forgetting in drosophila and rodents. DA, dopamine; DAMB, dopamine receptor in mushroom body (MB) neuron; D1/5, dopamine receptor subtype in mammals; DG, dentate gyrus; HP, hippocampus; PLC, phospholipase C. $\longrightarrow$ represents inhibition; $\rightarrow$ represents facilitation; arrows at the right part of neurons represent behavioral outcome.

acquisition or memory formation (Migues et al., 2016). Therefore, it was proposed that endocytosis of GluA2containing AMPA receptor in activated synapses appears to be one mechanisms of time-dependent forgetting in learning tasks with different valence. However, it is important to mention here that to fully accomplish predictions about an active forgetting mechanism it remains to be determined whether the facilitation of synaptic removal of GluA2-containing AMPA receptors induces forgetting of consolidated memories.
3. Rapid forgetting without impairments in memory formation or retrieval of long-lasting aversive/fear memories was consistently observed after inhibition of protein synthesis in the dorsal HP, amygdala, medial prefrontal, retrosplenial or insular cortices late after training (Bekinschtein et al., 2007; Ou et al., 2010; Martínez-Moreno et al., 2011; Katche et al., 2012; Gonzalez et al., 2013; Tomaiuolo et al., 2014). Twelve hours after training, BDNF was required and sufficient to sustain LTM storage for many days (Bekinschtein et al., 2007, 2008). In other words, BDNF attenuates forgetting. BDNF, via 
activation of $\operatorname{TrKB}$ receptors, ERK1/2 phosphorylation and c-fos, arc, and zif-268 expression promoted the establishment of a long-lasting fear LTM (Sweatt, 2001; Bekinschtein et al., 2008; Katche et al., 2010; Tomaiuolo et al., 2014; Nakayama et al., 2015, 2016). Inhibition of any of the molecular steps of this signaling cascade late after training induced rapid forgetting of LTM. More recently, the group of Alberini (Taubenfeld et al., 2001a; Bambah-Mukku et al., 2014) demonstrated that protein synthesis and BDNF signaling are required for at least $24 \mathrm{~h}$ after training to maintain avoidance memory storage in rats. Several afferent systems to the ventral tegmental area including those coming from the lateral habenula, the medial prefrontal cortex and the pedunculopontine tegmental nucleus may regulate temporal stability of consolidated fear LTM through the modulation of dopamine (DA)/BDNF signaling pathway in the HP (Lima et al., 2013; Gonzalez et al., 2014; Tomaiuolo et al., 2014).

What are the upstream neurotransmitters that trigger or modulate BDNF signaling to maintain long-lasting memories? DA in the dorsal HP has a critical role in the duration of LTM storage (Rossato et al., 2009). Long-lived fear memory vanished rapidly when the D1-DA receptor antagonist SCH23390 was injected into the dorsal HP $12 \mathrm{~h}$ after inhibitory avoidance training. On the other hand, delivery of the D1 agonist SK38393 at the same critical posttraining time converted a rapidly decaying fear LTM in a persistent one. NMDAr activation in the VTA upregulates the hippocampal dopaminergic system which through a D1-dependent mechanism controls the expression of BDNF required for LTM persistence (Rossato et al., 2009). Other extracellular signals that modulate the durability of memories via regulation of that late consolidation phase are noradrenaline (Katche et al., 2010; Mello-Carpes et al., 2016), serotonin (Slipczuk et al., 2013), glutamate (Rossato et al., 2009), acetylcholine (Parfitt et al., 2012; Porto et al., 2015), insulin-like growth factor 2 (Lee et al., 2015), spermidine (Signor et al., 2014), and the addictive drug nicotine (Lima et al., 2013).

4. Several genes or mechanisms that regulated transcription and translation have been also showed to selectively modify the duration of LTM without alterations in other stages of memory processing (Taubenfeld et al., 2001b. It has been reported that integrin beta 2 and Sterol O-acyltransferase 1 are two genes required for long-lasting contextual fear LTM tested 7 days after training, but not for shortlasting fear LTM tested 1 day posttraining (Matynia et al., 2008). Cytoplasmic polyadenylation element binding protein (CPEB), a molecule that activates dormant mRNAs, is required for the persistence but not formation of long-term facilitation (LTF) in Aplysia. A late phase of sustained CPEB prion-like multimer activity is also needed for the synaptic growth associated with persistent LTF in a local protein synthesis-dependent manner (Miniaci et al., 2008; Si et al., 2010). Genetic ablation of CPEB3 in mice induced active forgetting by impairing the maintenance of both hippocampal long-term potentiation and HP-dependent spatial memory (Fioriti et al., 2015).
Epigenetic mechanisms also contribute to modulate memory persistence and forgetting. It has been suggested that a shift in the regulatory balance activating NFkB transcription factor and histone acetylation is sufficient to render a memory more persistent (Federman et al., 2013). Briefly, inhibition of hippocampal histone acetyltransferases during consolidation of an object recognition task induced rapid forgetting while inhibition of hippocampal histone deacetylases, that normally silence transcription, induced persistent recognition memory (Stefanco et al., 2009; Federman et al., 2013). It has been also demonstrated that DNA methylation in the medial prefrontal cortex plays a critical role in the durability of fear memory (Miller et al., 2010). The authors found that a single contextual fear learning experience induced a persistent DNA hypermethylation in the medial prefrontal cortex. Intracortical inhibition of DNA methylation 1 month after training provoked forgetting, but not when the inhibitors were infused 1 day after conditioning (Miller et al., 2010).

Several studies reported changes associated with the process of forgetting. For instance, Tellez et al. (2012) found that forgetting is associated with modifications in some neural transporters in different brain regions including the hippocampus. It would be interesting to determine whether these neural transporters are required for active forgetting.

\section{DIRECT EVIDENCE FOR ACTIVE FORGETTING}

Although compelling and indirect evidence strongly support the hypothesis that nervous system has the property of actively erasing stored information, some direct evidence for the existence of such a function or mechanism in the brain is needed. Main predictions to consider direct evidence for active inherent forgetting is to find that the specific inhibition of that mechanism will promote memory storage maintenance and the selective stimulation of it will provoke forgetting. Some molecular mechanisms that become strong candidates to be part of active intrinsic forgetting like the synaptic removal of GluA2 receptors or the activity of some phosphatases (Genoux et al., 2002; Migues et al., 2010, 2016; Sachser et al., 2016) partially accomplish the above-mentioned predictions. In addition, both predictions need additional requirements in order to consider them appropriately fulfilled. Confounds factors like facilitation or inhibition of memory formation have to be experimentally ruled out in order to establish the existence of an inherent forgetting process. In my opinion, to avoid some confounds it is preferable to study intrinsic mechanisms of forgetting in consolidated memories. A good example of such requirements is the findings of GluA2 endocytosis in non-consolidated short-term inhibitory avoidance memory (Dong et al., 2015). Only when information endorsing both predictions and requirements is available, one can be sure that a mechanism of time-dependent active forgetting is present. To the best of our knowledge these predictions and requirements were totally achieved by a few molecular mechanisms in Drosophila and by one process in the rat (see below). 
In the following section I will review the scarce direct evidence regarding active inherent forgetting in brain circuits obtained so far. Some of the data mainly come from invertebrate (Drosophila) models of early unconsolidated memory, and the rest is provided by studies in consolidated memory in rodents. Therefore I will discuss first intrinsic mechanisms of active forgetting in Drosophila and then those observed in mice and rats.

\section{Active Forgetting in Drosophila}

Using a well-characterized olfactory aversive conditioning in Drosophila, the group of Zhong and colleagues (Shuai et al., 2010) showed that Rac1, a member of the Rho family of GTPases, is major player of active forgetting of early aversive memory. $\mathrm{Rac}$ is important for cytoskeleton dynamics and regulates synaptic spines through the modulation of actin polymerization (Heasman and Ridley, 2008). Inhibition of Rac1 induced a decrease in memory decay increasing its duration from a few hours to more than 1 day (see Figure 1). In contrast, stimulation of Rac activity induced forgetting of odor-shock association (Shuai et al., 2010). Therefore, Rac activity fulfills the main requisite to be considered a key step in active forgetting of early olfactory aversive memory in Drosophila. Hyperactivation of cofilin, a well-established downstream target of Rac1, enhanced early memory while inhibition of cofilin attenuated memory (Shuai et al., 2010).

Subsequently, the group of Ron Davis (Berry et al., 2012) reported that DA in the mushroom bodies of the fly has two functions: memory formation and forgetting. They studied $3 \mathrm{~h}$ memory retention of olfactory classical conditioning and found that a small subset of DA neurons, via posttraining activation of a DA receptor called dopamine receptor in mushroom body (DAMB) localized to intrinsic neurons of the mushroom bodies, are critical for active forgetting of early labile memory (see Figure 1). In addition, activation of these dopaminergic neurons induced forgetting of consolidated aversive as well as appetitive memories as tested at $6 \mathrm{~h}$ after training. DA via activation of another receptor $\mathrm{dDA} 1$ which is the Drosophila homolog of mammalian D1 receptor is required for aversive and appetitive memory acquisition (Kim et al., 2007). The authors proposed that after a new memory is formed both memory consolidation and active forgetting coexist, and that ongoing activity in a subset of dopaminergic neurons begins to erode what is being formed. Based on these considerations it is tempting to suggest that relevant or salient information to be stored enhances consolidation processes and/or attenuates active forgetting mechanisms.
Interestingly enough is that sleep after learning improves memory by blocking DA-based forgetting and arousal accelerates forgetting by increasing dopaminergic signaling due to sensory stimulation (Berry et al., 2015). It appears also that parallel systems exist that are involved in active forgetting: additional subset of DA cells and a couple of glutamatergic neurons were found to participate in olfactory aversive memory loss (Shuai et al., 2015). More recently it has been demonstrated that Scribble, a scaffolding protein that interacts with Rac1, Pak, and cofilin in mushroom body (MB) neurons, is required for forgetting of olfactory memories and is part of a signaling pathway triggered by DA acting on DAMB receptors (CervantesSandoval et al., 2016). These findings help understand how ongoing DA activity at $\mathrm{MB}$ neurons may regulate actin dynamics and cytoskeleton rearrangements to induce active forgetting.

Another member of Rho GTPases family is Cdc42. Importantly, it has been recently demonstrated that this small $\mathrm{G}$ protein is involved in active forgetting of consolidated olfactory aversive memory (Zhang et al., 2016). Conditioning activates this protein and induces forgetting without altering its formation while the expression of a dominant negative mutant of Cdc42 increases persistence of memory storage (see active forgetting in rodents). This finding highlights the idea that each phase of memory processing olfactory aversive conditioning in Drosophila is under the control of different forgetting mechanisms (Table 1).

\section{Active Forgetting in Rodents}

1. The role of Rac 1 in rodents is at least controversial. It is important to stress here that is difficult to compare the type and stages of memory processing in rodents with those in invertebrates (Davis, 2011). Only two recent works seem to study specifically whether this small G protein is involved in active forgetting. Liu et al. (2016) found that activation of Rac1 activity in hippocampal neurons accelerates forgetting of an object recognition task in mice: a memory that normally persists for more than $24 \mathrm{~h}$ but less than $72 \mathrm{~h}$ now with the expression of constitutive active Racl it is maintained less than 24 h. In contrast, inhibition of Racl activity prolongs the duration of object recognition memory up to 5 days (Figure 1). Unexpectedly, no changes in memory persistence were found in contextual fear conditioning and in trace fear conditioning (Liu et al., 2016). These findings suggest that Rac1-mediated forgetting is not a general mechanism of time-dependent memory loss.

TABLE 1 | Mechanisms of memory forgetting: behavioral effects of inhibition or facilitation of molecular events involved in active forgetting

\begin{tabular}{|c|c|c|c|c|}
\hline Animal target & Learning task & Memory stage & Inhibition & Facilitation \\
\hline Drosophila Rac1 & Olfactory aversive conditioning & Early & Persistence & Forgetting \\
\hline Drosophila Dopamine neurons & Olfactory aversive or appetitive conditioning & Early (3 h) & Persistence of early memory (6 h) & Forgetting \\
\hline Drosophila Dopamine receptor DAMB & Olfactory aversive conditioning & Early (3 h) & Persistence (Up to 24 h) & ND \\
\hline Drosophila scribble & Olfactory aversive conditioning & Early & Persistence (up to 24 h) & ND \\
\hline Drosophila Cdc42 & Olfactory aversive & Intermediate & Persistence of Anesthesia-resistant-memory & Forgetting \\
\hline Mouse Rac1 & Object recognition & LTM (24 h) & Increased duration (up to 5 days) & Forgetting \\
\hline Rat Dopamine receptor (D5?) & Conditioned place preference (appetitive) & LTM & Persistence of LTM (up to 14 days) & Forgetting \\
\hline Mouse Adult neurogenesis & Aversive and appetitive tasks & LTM & Persistence of LTM (up to 6 weeks) & Forgetting \\
\hline
\end{tabular}


Using massed and spaced contextual fear conditioning in rats Jiang et al. (2016) demonstrated that hippocampal inhibition of Rac1 with NSC27366 immediately after training facilitated memory retention in massed trained animals at 1 and 7 days, reaching similar freezing values to those obtained in spaced trained rats. The activation of Racl resulted in partial amnesia at the same time points. Together these findings suggest that Rac1 in the rat HP may be important for active forgetting of early fear memory. It would be interesting to determine whether inhibition of Racl affects immediate and short-term contextual fear memory.

Rac1 and its downstream effector cofilin also modulate 1-day cocaine-associated memory in conditioned place preference task. While activation of Racl in nucleus accumbens impaired memory, inhibition of Racl potentiated memory performance (Dietz et al., 2012). Unfortunately, this study did not include experiments to determine whether Racl is involved in active inherent forgetting to rule out the possibility that the abovementioned effects are merely due to modifications in the mechanisms of memory formation.

As it happened in Drosophila, it has been suggested that Cdc42 may participate in active forgetting. Cdc42 knockout mice exhibited normal acquisition and LTM retention performance in contextual fear conditioning and Morris water maze, but impaired remote memory recall (Kim et al., 2014). However, when a mechanism that induces forgetting is inhibited the main prediction is the increase in memory duration. Therefore, further experiments are needed to understand the role of $\mathrm{Cdc} 42$ in mammalian memory.

2. In the rat HP there are active molecular processes that fulfill the requirement to consider them as rapid inherent time-dependent forgetting mechanism. First, blocking hippocampal D1/D5 DA after learning induced a long-lasting cocaine-associated LTM extending the durability of the normally short-lasting LTM (single-trial conditioned place preference). This training is associated with delayed increase in DA levels in the dorsal HP (Kramar et al., 2014). In contrast, a D5 receptor agonist, but not a D1 receptor agonist, induced rapid forgetting of the normally long-lasting LTM (multipletrial conditioning; Kramar et al., 2014). The time-dependent memory loss effect on this positive-valence memory by dopaminergic stimulation of the dorsal HP is opposite to those effects obtained in two different negative-valence memories (inhibitory avoidance and conditioned place aversion): D1 receptor agonist induced BDNF-dependent persistence of LTM (from a short-lived memory that lasts a couple of days to a long-lived memory lasting more 2 weeks), whereas hippocampal D1/D5 receptor inhibition induced memory loss (Figure 1; Rossato et al., 2009; Kramar et al., 2014). Therefore, in both species Drosophila and rats DA is crucial for active time-dependent forgetting. While in Drosophila DA through the receptor DAMB is crucial for active forgetting of early non-consolidated memories of negative- and positive-experiences (Davis and Zhong, 2017), in rats two different hippocampal DA receptors, adenylyl cyclase-coupled D1 R and D5 R (phospholipase C
(PLC)-coupled D1 receptor), participate in active forgetting processes of negative and positive valence memories, respectively.

3. Finally, recent experimental evidence support the idea that adult neurogenesis in the HP bidirectionally regulates active natural forgetting (Figure 1). For instance, voluntary exercise during 6 weeks after fear conditioning induced an increase in neurogenesis and attenuated contextual fear conditioning and other HP-dependent learning tasks in mice such as inhibitory avoidance, water maze, Barnes maze and olfactory paired associate task (Akers et al., 2014; Epp et al., 2016). In contrast, inhibition of hippocampal neurogenesis decreased forgetting after a 6 week period following a water maze training (Epp et al., 2016). Together, these findings endorse the idea that adult neurogenesis in the HP play an important role in making room for new information (see Table $\mathbf{1}$ ).

\section{Active Mechanisms That Constrain Memory Formation}

In addition to intrinsic mechanisms of forgetting some other brain mechanisms may constrain the formation and consolidation of memories. The result of their blockade induces facilitation of memory retention and/or an increase in the duration of a given memory. Their stimulation may down-regulate memory strength or durability. Therefore, they appear not to be constituents of active inherent forgetting processes, but may well help limit the stability of new memories. The following are the best characterized constraints:

1. Calcineurin and protein phosphatase 1 are inhibitory constraints that normally down-regulated the formation and maintenance of nonassociative as well as associative learning tasks (Mansuy et al., 1998; Genoux et al., 2002; Baumgärtel et al., 2008). The expression of a calcineurin inhibitor enhances short-term and long-term object location and object recognition memories (Malleret et al., 2001). Acquisition and memory storage of a spatial training in a Morris water maze is also enhanced. In a similar way, positive valence memory is facilitated (Gerdjikov and Beninger, 2005). The level of calcineurin in the amygdala at the moment of aversive memory modulates the strength of a memory (Baumgärtel et al., 2008). Calcineurin may limit several key signaling pathways including $\mathrm{Ca}^{2+}$-dependent, adenylyl cyclases-dependent and protein kinases-dependent processes acting on ion channels, glutamate receptors, and transcription factors (Winder and Sweatt, 2001; Oliveria et al., 2007). Inhibition of PP1 prolongs remote, but not recent spatial memory when induced after learning, suggesting that PPI may promote active forgetting (Genoux et al., 2002). However, no experiments were done to establish whether activation of PPI is sufficient to active forgetting. A similar situation is established also with calcineurin (Sachser et al., 2016).

2. Chen et al. (2003) used transgenic mice with a dominantnegative inhibitor against the transcription factors ATF4 and $\mathrm{C} / \mathrm{EBP}$ and showed an increase in the duration of spatial memory switching a short-lived memory built up by using a 
weak training protocol to a long-lived spatial memory, which is consistent with the idea that these nuclear transcription factors are constraints of establishing remote memory. As previously shown, epigenetic modifications of histone deacetylase activity are constrains of consolidated LTM storage; inhibition of these enzymes in the dorsal HP caused weak objected recognition memory to persist (Federman et al., 2013).

\section{Processes Associated with Changes in the Rate of Forgetting}

1. Aging is commonly associated with rapid forgetting. Aged and young rodents have similar retention scores in many learning tasks when testing is about 1 day after training, but when testing occurs several days after aged rats and mice have poor memory (see for references Countryman and Gold, 2007). Rate of forgetting is associated with the activation of transcription factors like CREB. This is consistent with results obtained in spatial memory of aged mice with a genetic inhibition of PP1 that exhibit an increase in CREB transcriptional activity (Genoux et al., 2002).

2. Infantile amnesia. Natural forgetting of episodic, HP-dependent memories occurring during the first 3-4 years of our life (Hayne, 2004). A similar phenomenon is also present in other mammals, like rodents (Campbell and Spear, 1972). Both HP-dependent and independent memories acquired during a critical time period in the rat are rapidly forgotten (see for references Travaglia et al., 2016; Alberini and Travaglia, 2017). One of the two main hypotheses to explain this infantile amnesia is that during the first 3 years the rate of neurogenesis in the dentate gyrus (DG) of the hippocampal formation is very high compared to the adult brain and given the role of neurogenesis in forgetting (Feng et al., 2001; Akers et al., 2014), infantile amnesia is probable due to memory loss.

3. Acute stressful experiences during a critical late consolidation period after training bidirectionally regulate durability of fear-motivated HP-dependent LTM.

Cold water stress promoted the duration of short-lasting fear LTM from 1 day to more than 7 days (Yang et al., 2013). Administration of corticosterone had similar effects and the inhibitor of corticosterone synthesis metyrapone blocked stress and corticosterone-induced persistence of LTM. In addition, exposure to a novel, but not familiar, open field which rapidly elevates corticosterone levels (Handa et al., 1994), induced a long-lasting inhibitory avoidance memory in rats trained with a weak protocol that normally gives short-lasting LTM (Tomaiuolo et al., 2015). This

\section{REFERENCES}

Akers, K. G., Martinez-Canabal, A., Restivo, L., Yiu, A. P., De Cristofaro, A., Hsiang, H. L., et al. (2014). Hippocampal neurogenesis regulates forgetting in adulthood and infancy. Science 344, 598-602. doi: 10.1126/science. 1248903 promoting effect of spatial novelty on memory persistence is time-dependent, does not alter memory consolidation and requires D1/D5 DA receptors activation and Arc expression.

On the other hand, when we trained rats with strong inhibitory avoidance protocol that leaves long-lived avoidance LTM lasting 2-4 weeks, exposure to a novel environment induced rapid forgetting (Katche et al., 2016). Therefore, environmental factors can modify the duration of consolidated fear memory in a time-restricted manner.

\section{CONCLUDING REMARKS}

In the last 15 years emerges the idea that in addition to retroactive interference, retrieval-induced forgetting and passive decay of time (Anderson, 2003; Wixted, 2004), there are several processes in the nervous system of invertebrates and vertebrates that control what is stored and what is forgotten. These processes operate at different timescales and in different memory types. Some works immediately after acquisition at an early stage of memory formation and involves DA-releasing neurons, specific DA receptor subtypes, Racl activation, modifications in actin cytoskeleton at dendritic spines, and induces rapid active forgetting. Others regulate forgetting and persistence of seemingly consolidated memories modifying the activity of DA inputs to the HP and related brain regions activating parallel signaling cascades to induce active forgetting. Most of them may involve plastic changes in synaptic and extrasynaptic receptors including specific removal of GluA2 AMPA receptors. In addition, adult neurogenesis in the HP may induce forgetting at longer timescales. These are new and exciting examples of how brains are endowed with specific mechanisms to erase memory storage.

\section{AUTHOR CONTRIBUTIONS}

The author confirms being the sole contributor of this work and approved it for publication.

\section{FUNDING}

This study was supported by grants from Agencia Nacional de Promoción Científica y Tecnológica (ANPCyT, Argentina) to JHM; numbers: 2010-1169 and 2013-0335. Universidad de Buenos Aires (UBACyT, Argentina) 2014-2017 and the Argentina National Research Council (Consejo Nacional de Investigaciones Científicas y Técnicas, CONICET).
Alberini, C., and Travaglia, A. (2017). Infantile amnesia: a critical period of learning to learn and remember. J. Neurosci. 37, 5783-5795. doi: 10.1523/JNEUROSCI.0324-17.2017

Anderson, M. C. (2003). Rethinking interference theory: executive control and the mechanisms of forgetting. J. Mem. Lang. 49, 415-445. doi: 10.1016/j.jml.2003. 08.006 
Bambah-Mukku, D., Travaglia, A., Chen, D. Y., Pollonini, G., and Alberini, C. M. (2014). A positive autoregulatory BDNF feedback loop via C/EBP $\beta$ mediates hippocampal memory consolidation. J. Neurosci. 34, 12547-12559. doi: 10.1523/JNEUROSCI.0324-14.2014

Baumgärtel, K., Genoux, D., Welzl, H., Tweedie-Cullen, R. Y., Koshibu, K., Livingstone-Zatchej, M., et al. (2008). Control of the establishment of aversive memory by calcineurin and Zif268. Nat. Neurosci. 11, 572-578. doi: $10.1038 / \mathrm{nn} .2113$

Bekinschtein, P., Cammarota, M., Igaz, L. M., Bevilaqua, L. R., Izquierdo, I., and Medina, J. H. (2007). Persistence of long-term memory storage requires a late protein synthesis- and BDNF- dependent phase in the hippocampus. Neuron 53, 261-277. doi: 10.1016/j.neuron.2006.11.025

Bekinschtein, P., Cammarota, M., Katche, C., Slipczuk, L., Rossato, J. I., Goldin, A., et al. (2008). BDNF is essential to promote persistence of long-term memory storage. Proc. Natl. Acad. Sci. U S A 105, 2711-2716. doi: 10.1073/pnas. 0711863105

Bekinschtein, P., Cammarota, M., and Medina, J. H. (2014). BDNF and memory processing. Neuropharmacology 76, 677-683. doi: 10.1016/j.neuropharm.2013. 04.024

Berry, J. A., Cervantes-Sandoval, I., Chakraborty, M., and Davis, R. L. (2015). Sleep facilitates memory by blocking dopamine neuron-mediated forgetting. Cell 161, 1656-1667. doi: 10.1016/j.cell.2015.05.027

Berry, J. A., Cervantes-Sandoval, I., Nicholas, E. P., and Davis, R. L. (2012). Dopamine is required for learning and forgetting in Drosophila. Neuron 74, 530-542. doi: 10.1016/j.neuron.2012.04.007

Campbell, B. A., and Spear, N. E. (1972). Ontogeny of memory. Psychol. Rev. 79, 215-236. doi: $10.1037 / \mathrm{h} 0032690$

Cervantes-Sandoval, I., Chakraborty, M., MacMullen, C., and Davis, R. L. (2016). Scribble scaffolds a signalosome for active forgetting. Neuron 90, 1230-1242. doi: 10.1016/j.neuron.2016.05.010

Chen, A., Muzzio, I. A., Malleret, G., Bartsch, D., Verbitsky, M., Pavlidis, P., et al. (2003). Inducible enhancement of memory storage and synaptic plasticity in transgenic mice expressing an inhibitor of ATF4 (CREB-2) and C/EBP proteins. Neuron 39, 655-669. doi: 10.1016/s0896-6273(03) 00501-4

Countryman, R. A., and Gold, P. E. (2007). Rapid forgetting of social transmission of food preferences in aged rats: relationship to hippocampal CREB activation. Learn. Mem. 14, 350-358. doi: 10.1101/lm.524907

Cui, Z., Wang, H., Tan, Y., Zaia, K. A., Zhang, S., and Tsien, J. Z. (2004). Inducible and reversible NR1 knockout reveals crucial role of the NMDA receptor in preserving remote memories in the brain. Neuron 41, 781-793. doi: $10.1016 / \mathrm{s} 0896-6273(04) 00072-8$

Davis, R. L. (2011). Traces of Drosophila memory. Neuron 70, 8-19. doi: 10.1016/j. neuron.2011.03.012

Davis, R. L., and Zhong, Y. (2017). The biology of forgetting. Neuron 95, 490-503. doi: 10.1016/j.neuron.2017.05.039

Dietz, D. M., Sun, H., Lobo, M. K., Cahill, M. E., Chadwick, B., Gao, V., et al. (2012). Racl is essential in cocaine-induced structural plasticity of nucleus accumbens neurons. Nat. Neurosci. 15, 891-896. doi: 10.1038/ nn.3094

Dong, Z., Han, H., Li, H., Bai, Y., Wang, W., Tu, M., et al. (2015). Long-term potentiation decay and memory loss are mediated by AMPAR endocytosis. J. Clin. Invest. 125, 234-247. doi: 10.1172/JCI77888

Dudai, Y. (2002). Molecular bases of long-term memories: a question of persistence. Curr. Opin. Neurobiol. 12, 211-216. doi: 10.1016/s09594388(02)00305-7

Eckel-Mahan, K. L. (2012). Circadian oscillations within the hippocampus support memory formation and persistence. Front. Mol. Neurosci. 5:46. doi: $10.3389 /$ fnmol.2012.00046

Eckel-Mahan, K. L., Phan, T., Han, S., Wang, H., Chan, G. C.-K., Scheiner, Z. S., et al. (2008). Circadian oscillation of hippocampal MAPK activity and cAmp: implications for memory persistence. Nat. Neurosci. 11, 1074-1082. doi: $10.1038 / \mathrm{nn} .2174$

Epp, J. R., Silva Mera, R., Köhler, S., Josselyn, S. A., and Frankland, P. W. (2016). Neurogenesis-mediated forgetting minimizes proactive interference. Nat. Commun. 7:10838. doi: 10.1038/ncomms10838

Federman, N., de la Fuente, V., Zalcman, G., Corbi, N., Onori, A., Passananti, C., et al. (2013). Nuclear factor $\kappa \mathrm{B}$-dependent histone acetylation is specifically involved in persistent forms of memory. J. Neurosci. 33, 7603-7614. doi: 10.1523/JNEUROSCI.4181-12.2013

Feng, R., Rampon, C., Tang, Y.-P., Shrom, D., Jin, J., Kyin, M., et al. (2001). Deficient neurogenesis in forebrain-specific presenilin-1 knockout mice is associated with reduced clearance of hippocampal memory traces. Neuron 32, 911-926. doi: 10.1016/s0896-6273(01)00523-2

Fioriti, L., Myers, C., Huangg, Y.-Y., Li, X., Stephan, J. S., Trifilieff, P., et al. (2015). The persistence of hippocampal-based memory requires protein synthesis mediated by the prion-like protein CPEB3. Neuron 86, 1433-1448. doi: 10.1016/j.neuron.2015.05.021

Frankland, P. W., Köhler, S., and Josselyn, S. (2013). Hippocampal neurogenesis and forgetting. Trends Neurosci. 36, 497-503. doi: 10.1016/j.tins.2013. 05.002

Frankland, P. W., O’Brien, C., Ohno, M., Kirkwood, A., and Silva, A. J. (2001). $\alpha$-CaMKII-dependent plasticity in the cortex is required for permanent memory. Nature 411, 309-313. doi: 10.1038/35077089

Genoux, D., Haditsch, U., Knobloch, M., Michalon, A., Storm, D., and Mansuy, I. M. (2002). Protein phosphatase 1 is a molecular constraint of learning and memory. Nature 418, 970-975. doi: 10.1038/nature00928

Gerdjikov, T. V., and Beninger, R. J. (2005). Differential effects of calcineurin inhibition and protein kinase $\mathrm{A}$ activation on nucleus accumbens amphetamine-produced conditioned place preference in rats. Eur. J. Neurosci. 22, 697-705. doi: 10.1111/j.1460-9568.2005.04256.x

Gonzalez, C., Kramar, C., Garagoli, F., Rossato, J. I., Weisstaub, N., Cammarota, M., et al. (2013). Medial prefrontal cortex is a crucial node of a rapid learning system that retrieves recent and remote memories Neurobiol. Learn. Mem. 103, 19-25. doi: 10.1016/j.nlm.2013.04.006

Gonzalez, M. C., Kramar, C., Tomaiuolo, M., Katche, C., Weisstaub, N., Cammarota, M., et al. (2014). Medial prefrontal cortex dopamine controls the persistent storage of aversive memories. Front. Behav. Neurosci. 8:408. doi: 10.3389/fnbeh.2014.00408

Handa, R. J., Nunley, K. M., Lorens, S. A., Louie, J. P., McGivern, R. F., and Bollnow, M. R. (1994). androgen regulation of adrenocorticotropin and corticosterone secretion in the male rat following novelty and foot shock stressors. Physiol. Behav. 55, 117-124. doi: 10.1016/0031-9384(94)90 018-3

Hardt, O., Nader, K., and Nadel, L. (2013). Decay happens: the role of active forgetting in memory. Trends Cogn. Sci. 17, 111-120. doi: 10.1016/j.tics.2013. 01.001

Hayne, H. (2004). Infant memory development: implications for childhood amnesia. Dev. Rev. 24, 33-73. doi: 10.1016/j.dr.2003.09.007

Heasman, S. J., and Ridley, A. J. (2008). Mammalian Rho GTPases: new insights into their functions from in vivo studies. Nat. Rev. Mol. Cell Biol. 9, 690-701. doi: $10.1038 / \mathrm{nrm} 2476$

Jiang, L., Mao, R., Zhou, Q., Yang, Y., Cao, J., Ding, Y., et al. (2016). Inhibition of Racl activity in the hippocampus impairs the forgetting of contextual fear memory. Mol. Neurobiol. 53, 1247-1253. doi: 10.1007/s12035-015-9093-6

Kandel, E. R. (2001). The molecular biology of memory storage: a dialogue between genes and synapses. Science 294, 1030-1038. doi: 10.1126/science. 1067020

Katche, C., Bekinschtein, P., Slipczuk, L., Goldin, A., Izquierdo, I. A., Cammarota, M., et al. (2010). Delayed wave of c-Fos expression in the dorsal hippocampus involved specifically in persistence of long-term memory storage. Proc. Natl. Acad. Sci. U S A 107, 349-354. doi: 10.1073/pnas.0912931107

Katche, C., Cammarota, M., and Medina, J. H. (2013a). Molecular signatures and mechanisms of long-lasting memory consolidation and storage. Neurobiol. Learn. Mem. 106, 40-47. doi: 10.1016/j.nlm.2013.06.018

Katche, C., Dorman, G., Gonzalez, M. C., Kramar, C. P., Slipczuk, L., Rossato, J. I., et al. (2013b). On the role of retrosplenial cortex in long-lasting memory storage. Hippocampus 23, 295-302. doi: 10.1002/hipo.22092

Katche, C., Goldin, A., Gonzalez, C., Bekinschtein, P., and Medina, J. H. (2012). Maintenance of long-term memory storage is dependent on late posttraining Egr-1 expression. Neurobiol. Learn. Mem. 98, 220-227. doi: 10.1016/j.nlm.2012. 08.001

Katche, C., and Medina, J. H. (2017). Requirement of an early activation of $\mathrm{BDNF} / \mathrm{c}-\mathrm{Fos}$ cascade in the retrosplenial cortex for the persistence of a long-lasting aversive memory. Cereb. Cortex 27, 1060-1067. doi: $10.1093 /$ cercor/bhv284 
Katche, C., Tomaiuolo, M., Dorman, G., Medina, J. H., and Viola, H. (2016). Novelty during a late postacquisition time window attenuates the persistence of fear memory. Sci. Rep. 6:e35220. doi: 10.1038/srep35220

Kim, Y. C., Lee, H. G., and Han, K. A. (2007). $\mathrm{D}_{1}$ dopamine receptor dDA1 is required in the mushroom body neurons for aversive and appetitive learning in Drosophila. J. Neurosci. 27, 7640-7647. doi: 10.1523/JNEUROSCI.1167 $-07.2007$

Kim, I. H., Wang, H., Soderling, S. H., and Yasuda, R. (2014). Loss of Cdc42 leads to defects in synaptic plasticity and remote memory recall. Elife 3:e02839. doi: $10.7554 /$ eLife.02839

Kramar, C. P., Chefer, V. I., Wise, R. A., Medina, J. H., and Barbano, M. F. (2014). Dopamine in the dorsal hippocampus impairs the late consolidation of cocaine-associated memory. Neuropsychopharmacology 39, 1645-1653. doi: $10.1038 / n p p .2014 .11$

Lee, Y., Lee, Y. G., Gao, G., Lee, L., Lee, H. E., and Rhu, J. H. (2015). Exogenous insulin-like growth factor 2 administration enhances memory consolidation and persistence in a time-dependent manner. Brain Res. 1622, 466-473. doi: 10.1016/j.brainres.2015.07.002

Lima, R. H., Radiske, A., Köhler, C. A., Gonzalez, M. C., Bevilaqua, L. R., Rossato, J. I., et al. (2013). Nicotine modulates the long-lasting storage of fear memory. Learn. Mem. 20, 120-124. doi: 10.1101/lm.029900.112

Liu, Y., Du, S., Lv, L., Lei, B., Shi, W., Tang, Y., et al. (2016). Hippocampal activation of Rac1 regulates the forgetting of object-recognition memory. Curr. Biol. 26, 2351-2357. doi: 10.1016/j.cub.2016.06.056

Malleret, G., Haditsch, U., Genoux, D., Jones, M. W., Bliss, T. V., Vanhoose, A. M., et al. (2001). Inducible nad reversible enhancement of learning, memory, and LTP by genetic inhibition of calcineurin. Cell 140, 675-686. doi: 10.1016/s00928674(02)08015-7

Mansuy, I. M., Mayford, M., Jacob, B., Kandel, E. R., and Bach, M. E. (1998). Restricted and regulated overexpression reveals calcineurin as a key component in the transition from short-term to long-term memory. Cell 92, 39-49. doi: 10.1016/s0092-8674(00)80897-1

Martínez, M. C., Allen, N., Ballarini, F., Moncada, D., and Viola, H. (2012). Memory traces compete under regimes of limited Arc protein synthesis: implications for memory interference. Neurobiol. Learn. Mem. 98, 165-173. doi: 10.1016/j.nlm.2012.05.007

Martínez-Moreno, A., Rodríguez-Durán, L. F., and Escobar, M. L. (2011). Late protein synthesis-dependent phases in CTA long-term memory: BDNF requirement. Front. Behav. Neurosci. 5:61. doi: 10.3389/fnbeh.2011.00061

Matynia, A., Anagnostaras, S., Witgen, B. J., Lacuesta, M., Fanselow, M. S., and Silva, A. J. (2008). A high through-put reverse genetic screen identifies two genes involved in remote memory in mice. PLoS One 3:e2121. doi: 10.1371 /journal.pone. 0002121

McGaugh, J. L. (2000). Memory-a century of consolidation. Science 287, 248-251. doi: $10.1126 /$ science.287.5451.248

Mello-Carpes, P. B., da Silva de Vargas, L., Gayer, M. C., Roehrs, R., and Izquierdo, I. (2016). Hippocampal noradrenergic activation is necessary for object recognition memory consolidation and can promote BDNF increase and memory persistence. Neurobiol. Learn. Mem. 127, 84-92. doi: 10.1016/j.nlm. 2015.11.014

Migues, P. V., Hardt, O., Wu, D. C., Gamache, K., Sacktor, T. C., Wang, Y. T., et al. (2010). PKMzeta maintains memories by regulating GluR2-dependent AMPA receptor trafficking. Nat. Neurosci. 13, 630-634. doi: 10.1038/nn.2531

Migues, P. V., Liu, L., Archbold, G. E., Einarsson, E. Ö., Wong, J., Bonasia, K., et al. (2016). Blocking synaptic removal of GluA2-containing AMPA receptors prevents the natural forgetting of long term memories. J. Neurosci. 36, 3481-3494. doi: 10.1523/JNEUROSCI.3333-15.2016

Miller, C. A., Gavin, C. F., White, J. A., Parrish, R. R., Honasoge, A., Yancey, C. R., et al. (2010). Cortical DNA methylation maintains remote memory. Nat. Neurosci. 13, 664-666. doi: 10.1038/nn.2560

Miniaci, M. C., Kim, J. H., Puthanveettil, S. V., Si, K., Zhu, H., Kandel, E. R., et al. (2008). Sustained CPEB-dependent local protein synthesis is required to stabilize synaptic growth for persistence of long-term facilitation in Aplysia. Neuron 59, 1024-1036. doi: 10.1016/j.neuron.2008.07.036

Morris, R. G. (2006). Elements of a neurobiological theory of hippocampal function: the role of synaptic plasticity, synaptic tagging and schemas. Eur. J. Neurosci. 23, 2829-2846. doi: 10.1111/j.1460-9568.2006. 04888.x
Nakayama, D., Hashikawa-Yamasaki, Y., Ikegaya, Y., Matsuki, N., and Nomura, H. (2016). Late Arc/Arg3.1 expression in the basolateral amygdala is essential for persistence of newly-acquired and reactivated contextual fear memories. Sci. Rep. 16:21007. doi: 10.1038/srep21007

Nakayama, D., Iwata, H., Teshirogi, C., Ikegaya, Y., Matsuki, N., and Nomura, H. (2015). Long delayed expression of the immediate early gene Arc/Arg3.1 refines the neuronal circuits to perpetuate fear memory. J. Neurosci. 35, 819-830. doi: 10.1523/JNEUROSCI.2525-14.2015

Neath, I., and Brown, G. D. A. (2012). Arguments against memory trace decay: a simple account of Baddeley and Scott. Front. Psychol. 3:35. doi: 10.3389/fpsyg. 2012.00035

Oliveria, S. F., Dell'Acqua, M. L., and Sather, W. A. (2007). AKAP79/150 anchoring of calcineurin controls neuronal L-type $\mathrm{Ca}^{2+}$ channel activity and nuclear signaling. Neuron 55, 261-275. doi: 10.1016/j. neuron.2007.06.032

Ou, L. C., Yeh, S. H., and Gean, P. W. (2010). Late expression of brain-derived neurotrophic factor in the amygdala is required for persistence of fear memory. Neurobiol. Learn. Mem. 93, 372-382. doi: 10.1016/j.nlm.2009.12.003

Pan, Y. W., Chan, G. C., Kuo, C. T., Storm, D. R., and Xia, Z. (2012). Inhibition of adult neurogenesis by inducible and targeted deletion of ERK5 mitogenactivated protein kinase specifically in adult neurogenic regions impairs contextual fear extinction and remote fear memory. J. Neurosci. 32, 6444-6455. doi: 10.1523/JNEUROSCI.6076-11.2012

Parfitt, G. M., Campos, R. C., Barbosa, A. K., Koth, A. P., and Barros, D. M. (2012). Participation of hippocampal cholinergic system in memory persistence for inhibitory avoidance in rats. Neurobiol. Learn. Mem. 97, 183-188. doi: 10.1016/j.nlm.2011.12.001

Porto, G. P., Milanesi, L. H., Rubin, M. A., and Mello, C. F. (2015). Effect of morphine on the persistence of long-term memory in rats. Psychopharmacology (Berl) 232, 1747-1753. doi: 10.1007/s00213-014-3811-z

Rossato, J. I., Bevilaqua, L. R., Izquierdo, I., Medina, J. H., and Cammarota, M. (2009). Dopamine controls persistence of long-term memory storage. Science 325, 1017-1020. doi: 10.1126/science.1172545

Sachser, R., Santana, F., Crestani, A., Lunardi, P., Pedraza, L. K., Quillfeldt, J. A., et al. (2016). Forgetting of long-term memory requires activation of NMDA receptors, L-type voltage-dependent $\mathrm{Ca}^{2+}$ channels and calcineurin. Sci. Rep. 6:22771. doi: $10.1038 /$ srep22771

Sacktor, T. C. (2011). How does PKM $\zeta$ maintain long-term memory? Nat. Rev. Neurosci. 12, 9-15. doi: 10.1038/nrn2949

Sara, S. J. (2000). Retrieval and reconsolidation: toward a neurobiology of remembering. Learn. Mem. 7, 73-84. doi: 10.1101/lm.7.2.73

Shan, Q., Chan, G. C., and Storm, D. R. (2008). Type 1 adenylyl cyclase is essential for maintenance of remote contextual fear memory. J. Neurosci. 28, 12864-12867. doi: 10.1523/JNEUROSCI.2413-08.2008

Shuai, Y., Hirokawa, A., Ai, Y., Zhang, M., Li, W., and Zhong, Y. (2015). Dissecting neural pathways for forgetting in Drosophila olfactory aversive memory. Proc Natl. Acad. Sci. U S A 112, E6663-E6672. doi: 10.1073/pnas.1512792112

Shuai, Y., Lu, B., Hu, Y., Wang, L., Sun, K., and Zhong, Y. (2010). Forgetting is regulated through Rac activity in Drosophila. Cell 140, 579-589. doi: 10.1016/j. cell.2009.12.044

Si, K., Choi, Y. B., White-Grindley, E., Majumdar, A., and Kandel, E. R. (2010). Aplysia CPEB can form prion-like multimers in sensory neurons that contribute to long-term facilitation. Cell 140, 421-435. doi: 10.1016/j.cell.2010. 01.008

Signor, C., Mello, C. F., Porto, G. P., Ribeiro, D. A., and Rubin, M. A. (2014). Spermidine improves fear memory persistence. Eur. J. Pharmacol. 730, 72-76. doi: 10.1016/j.ejphar.2014.02.035

Skaggs, E. B. (1933). A discussion on the temporal point of interpolation and degree of retroactive inhibition. J. Comp. Psychol. 16, 411-414. doi: $10.1037 / \mathrm{h} 0072751$

Slipczuk, L., Tomaiuolo, M., Garagoli, F., Weisstaub, N., Katche, C., Bekinschtein, P., et al. (2013). Attenuating the persistence of fear memory storage using a single dose of antidepressant. Mol. Psychiatry 18, 7-8. doi: $10.1038 / \mathrm{mp} .2012 .4$

Stefanco, D. P., Barrett, R. M., Ly, A. R., Reolon, G. K., and Wood, M. (2009). Modulation of long-term memory for object recognition via HDAC inhibition. Proc. Natl. Acad. Sci. U S A 106, 9447-9452. doi: 10.1073/pnas.09039 64106 
Sweatt, J. D. (2001). Memory mechanisms: the yin and yan of protein phosphoylation. Curr. Biol. 11, R391-R394. doi: 10.1016/s0960-9822(01) 00216-0

Taubenfeld, S. M., Milekic, M. H., Monti, B., and Alberini, C. M. (2001a). The consolidation of new but not reactivated memory requires hippocampal C/EBP $\beta$. Nat. Neurosci. 4, 813-818. doi: 10.1038/90520

Taubenfeld, S. M., Wiig, K. A., Monti, B., Dolan, B., Pollonini, G., and Alberini, C. M. (2001b). Fornix-dependent induction of hippocampal CCAAT enhancer-binding protein $\beta$ and $\delta$ Co-localizes with phosphorylated cAMP response element-binding protein and accompanies long-term memory consolidation. J. Neurosci. 21, 84-91.

Tellez, R., Gómez-Viquez, L., Liy-Salmeron, G., and Meneses, A. (2012). GABA, glutamate, dopamine and serotonin transporters expression on forgetting. Neurobiol. Learn. Mem. 98, 66-77. doi: 10.1016/j.nlm.2012.05.001

Tomaiuolo, M., Gonzalez, M. C., Medina, J. H., and Piriz, J. (2014). The lateral habenula determines long-term storage of aversive memories. Front. Behav. Neurosci. 8:170. doi: 10.3389/fnbeh.2014.00170

Tomaiuolo, M., Katche, C., Viola, H., and Medina, J. H. (2015). Evidence of maintenance tagging in the hippocampus for the persistence of long-term memory storage. Neural Plast. 2015:603672. doi: 10.1155/2015/603672

Travaglia, A., Bisaz, R., Sweet, E. S., Blitzer, R. D., and Alberini, C. M. (2016). Infantile amnesia reflects a developmental critical period for hippocampal learning. Nat. Neurosci. 19, 1225-1233. doi: 10.1038/nn.4348

Wang, H., Shimizu, E., Tang, Y. P., Cho, M., Kyin, M., Zuo, W., et al. (2003). Inducible protein knockout reveals temporal requirement of CaMKII reactivation for memory consolidation in the brain. Proc. Natl. Acad. Sci. US A 100, 4287-4292. doi: 10.1073/pnas.0636870100
Wieczorek, L., Maas, J. W. Jr., Muglia, L. M., Vogt, S. K., and Muglia, L. J. (2010). Temporal and regional regulation of gene expression by calciumstimulated adenylyl cyclase activity during fear memory. PLoS One 5:e13385. doi: 10.1371/journal.pone.0013385

Winder, G. D., and Sweatt, J. D. (2001). Roles of serine/threonine phosphatases in hippocampal synaptic plasticity. Nat. Rev. Neurosci. 2, 461-474. doi: $10.1038 / 35081514$

Wixted, J. T. (2004). The psychology and neuroscience of forgetting. Annu. Rev. Psychol. 55, 235-269. doi: 10.1146/annurev.psych.55.090902.141555

Yang, C., Liu, J. F., Chai, B. S., Fang, Q., Chai, N., Zhao, L. Y., et al. (2013). Stress within a restricted time window selectively affects the persistence of long-term memory. PLoS One 8:e59075. doi: 10.1371/journal.pone. 0059075

Zhang, X., Li, Q., Wang, L., Liu, Z. J., and Zhong, Y. (2016). Cdc42-dependent forgetting regulates repetition effect in prolonging memory retention. Cell Rep. 16, 817-825. doi: 10.1016/j.celrep.2016.06.041

Conflict of Interest Statement: The author declares that the research was conducted in the absence of any commercial or financial relationships that could be construed as a potential conflict of interest.

Copyright (C) 2018 Medina. This is an open-access article distributed under the terms of the Creative Commons Attribution License (CC BY). The use, distribution or reproduction in other forums is permitted, provided the original author(s) and the copyright owner are credited and that the original publication in this journal is cited, in accordance with accepted academic practice. No use, distribution or reproduction is permitted which does not comply with these terms. 